\title{
Rad9a is involved in chromatin decondensation and post-zygotic embryo development in mice
}

\author{
Lin Huang ${ }^{1,2} \cdot$ Tie-Gang Meng ${ }^{1,3} \cdot$ Xue-Shan Ma ${ }^{1,4} \cdot$ Zhen-Bo Wang $^{1,3} \cdot$ Shu-Tao Qi ${ }^{1} \cdot$ Qi Chen ${ }^{1} \cdot$ Qing-Hua Zhang ${ }^{1}$. \\ Qiu-Xia Liang ${ }^{1,3} \cdot$ Zhong-Wei Wang $^{1} \cdot$ Meng-Wen $\mathrm{Hu}^{1} \cdot$ Lei Guo $^{1} \cdot$ Ying-Chun Ouyang ${ }^{1} \cdot \mathrm{Yi} \mathrm{Hou}^{1} \cdot$ Yong Zhao $\mathbb{D}^{5}$. \\ Qing-Yuan Sun ${ }^{1,3}$
}

Received: 22 March 2018 / Revised: 1 July 2018 / Accepted: 20 July 2018 / Published online: 28 August 2018

(c) ADMC Associazione Differenziamento e Morte Cellulare 2018

\begin{abstract}
Zygotic chromatin undergoes extensive reprogramming immediately after fertilization. It is generally accepted that maternal factors control this process. However, little is known about the underlying mechanisms. Here we report that maternal RAD9A, a key protein in DNA damage response pathway, is involved in post-zygotic embryo development, via a mouse model with conditional depletion of Rad9a alleles in oocytes of primordial follicles. Post-zygotic losses originate from delayed zygotic chromatin decondensation after depletion of maternal RAD9A. Pronucleus formation and DNA replication of most mutant zygotes are therefore deferred, which subsequently trigger the G2/M checkpoint and arrest development of most mutant zygotes. Delayed zygotic chromatin decondensation could also lead to increased reabsorption of postimplantation mutant embryos. In addition, our data indicate that delayed zygotic chromatin decondensation may be attributed to deferred epigenetic modification of histone in paternal chromatin after fertilization, as fertilization and resumption of secondary meiosis in mutant oocytes were both normal. More interestingly, most mutant oocytes could not support development beyond one-cell stage after parthenogenetic activation. Therefore, RAD9A may also play an important role in maternal chromatin reprogramming. In summary, our data reveal an important role of RAD9A in zygotic chromatin reprogramming and female fertility.
\end{abstract}

These authors contributed equally: Lin Huang, Tie-Gang Meng, XueShan Ma, Zhen-Bo Wang.

Edited by M Piacetini.

Electronic supplementary material The online version of this article (https://doi.org/10.1038/s41418-018-0181-9) contains supplementary material, which is available to authorized users.

Qing-Yuan Sun

sunqy@ioz.ac.cn

1 State Key Laboratory of Stem Cell and Reproductive Biology, Institute of Zoology, Chinese Academy of Sciences, Beijing, China

2 Department of Pediatrics, The Affiliated Hospital of Southwest Medical University, Luzhou, Sichuan, China

3 University of Chinese Academy of Sciences, Beijing, China

4 The Reproductive Medical Center, the First Affiliated Hospital, Zhengzhou University, Zhengzhou 450052, China

5 State Key Laboratory of Biomembrane, Institute of Zoology, Chinese Academy of Sciences, Beijing, China

\section{Introduction}

In mammalian zygotes, chromatin undergoes drastic reprogramming after fertilization. It includes protaminehistone replacement, chromatin decondensation, histone modifications, and so on $[1,2]$. It is widely accepted that maternal factors mainly control chromatin-reprogramming process [3]. Indeed, totipotency of somatic cells can be fully recovered via transferring their nuclei into the oocytes [4]. And studies have started to uncover the underlying mechanisms about protamine-histone replacement in mice [5]. There are also data implying the importance of maternal genome reprogramming in zygote development [5]. Maternal genome also drastically loses higher-order structure after fertilization [6]. However, we need more efforts to elaborate the factors in zygotic chromatin reprogramming.

Cells have developed complex DNA damage response pathways to maintain genomic stability. ATR and ATM signaling pathways are the two main DNA damage response pathways in eukaryotic cells [7,8]. To maintain genomic 
Fig. 1 Location of RAD9A during pre-zygotic and postzygotic embryo development. a Immunohistochemistry analysis showing the location of RAD9A in follicles at different stages. ProF, primordial follicles; PriF, primary follicles; $\mathrm{SecF}$, secondary follicles. Bar, $100 \mu \mathrm{m}$. b Confocal microscopy showing the subcellular location of RAD9A in mouse oocytes at different stages. Bar, $20 \mu \mathrm{m}$. c Confocal microscopy showing the subcellular location of RAD9A in mouse embryos. Bar, $20 \mu \mathrm{m}$. d Confocal microscopy confirming the subcellular location of RAD9A after myctagged RAD9A mRNA microinjection and anti MycFITC antibody staining in mouse oocytes at different stages. Bar, $20 \mu \mathrm{m}$
A

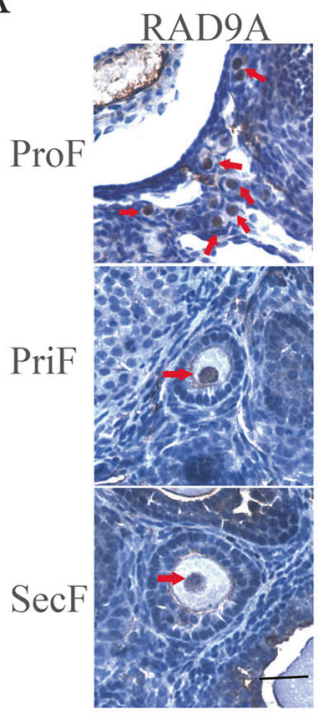

B

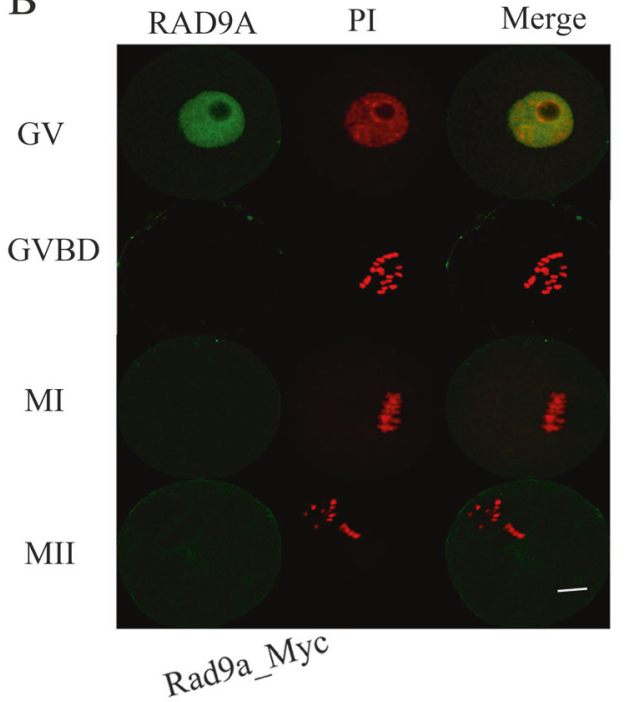

D PI Merge

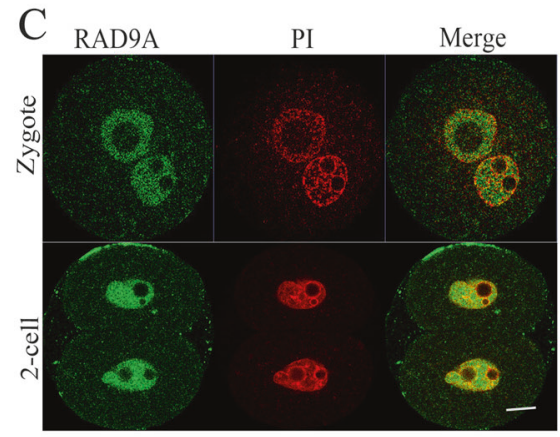

integrity, members in DNA damage response pathways work coordinately to detect and repair damage sites. They need to sense various aberrations of DNA structural alterations and respond properly $[9,10]$. This implies that proteins in DNA damage response pathways may be potential candidates of maternal factors in zygotic chromatin reprogramming.

As a key factor in DNA damage response pathways, mouse $\operatorname{Rad} 9 a$ gene is highly conserved during evolution [11]. In mammalian cells, RAD9A functions always as part of the heterotrimeric 9-1-1 complex formed by RAD9A, RAD1, and HUS1 [12]. RAD9A primarily participates in various mitotic DNA repair process, recruited by both ATR and ATM signaling pathways [7, 8]. RAD9A recruits the phosphorylated form of histone $\mathrm{H} 2 \mathrm{~A}$ variant $\mathrm{X}(\gamma \mathrm{H} 2 \mathrm{AX})$ to DNA damage sites. And inactivated RAD9A delays the appearance and disappearance of ionizing radiationinduced $\gamma \mathrm{H} 2 \mathrm{AX}$ foci, implying that RAD9A may participate in the modulation of chromatin structure $[13,14]$. It is also verified that $\operatorname{Rad} 9 a$ has a pivotal role in repair of meiotic DNA double-strand breaks (DSBs) during spermatogenesis in mice [15]. In addition to its well-known function in DNA damage response, mammalian RAD9A can also work as a regulating transcription factor [16]. $\operatorname{Rad} 9 a$ gene is also important for maintenance of chromosomal stability [13, 14]. Human cells with overexpressed RAD9A protein show programmed death [17, 18]. Our recent study also demonstrated that RAD9A has an antiapoptosis function during spermatogonia differentiation [19]. Moreover, RAD9A protein was also reported to serve as an oncongene or tumor suppressor [20-23]. Global disruption of RAD9A leads to lethality of mouse embryos, and even heterozygous $\operatorname{Rad} 9 a$ knockout mice are susceptive to cataractogenesis $[24,25]$.

Here in this study, we investigated the effect of conditional ablation of $\operatorname{Rad} 9 a$ alleles in oocytes on female reproductive activity and the underlying mechanisms, via crossing $\operatorname{Rad} 9 a^{f l o x / f l o x}$ mice (referred to as $\operatorname{Rad} 9 a^{F / F}$ ) with transgenic mice expressing growth differentiation factor 9 promoter-mediated Cre recombinase (referred to as GCre mice) $[24,26,27]$. The female mice with oocyte-specific disruption of RAD9A were severely subfertile. We 
Fig. 2 RAD9A is pivotal for fertility of female mice. a Schematics of specific deletion of $\operatorname{Rad} 9 a$ gene driven by $G d f 9$ Cre. b Oocyte-specific ablation of RAD9A verified by immunohistochemistry analysis of the ovaries of both genotypes. Bar, $100 \mu \mathrm{m}$. c Western blot analysis confirming the ablation of RAD9A in the $\operatorname{Rad} 9 a^{F / F}$; GCre oocytes, using GAPDH as a loading control. d $\operatorname{Rad} 9 a^{F / F}$; GCre female mice gave birth to much fewer pups than $\operatorname{Rad} 9 a^{F / F}$ control mice. e The litter size of $\operatorname{Rad} 9 a^{F / F} ;$ GCre female mice was much smaller than that of $\operatorname{Rad} 9 a^{F / F}$ mice. $*, P<0.05$. f $\operatorname{Rad} 9 a^{F / F} ;$ GCre female mice had less litters than $\operatorname{Rad} 9 a^{F / F}$ mice. *, $P<0.05$
A

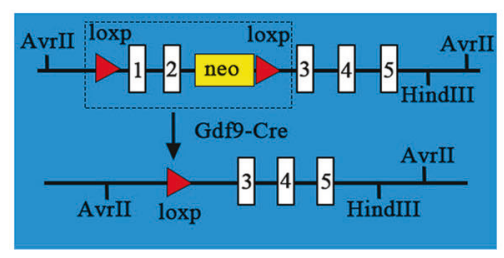

B
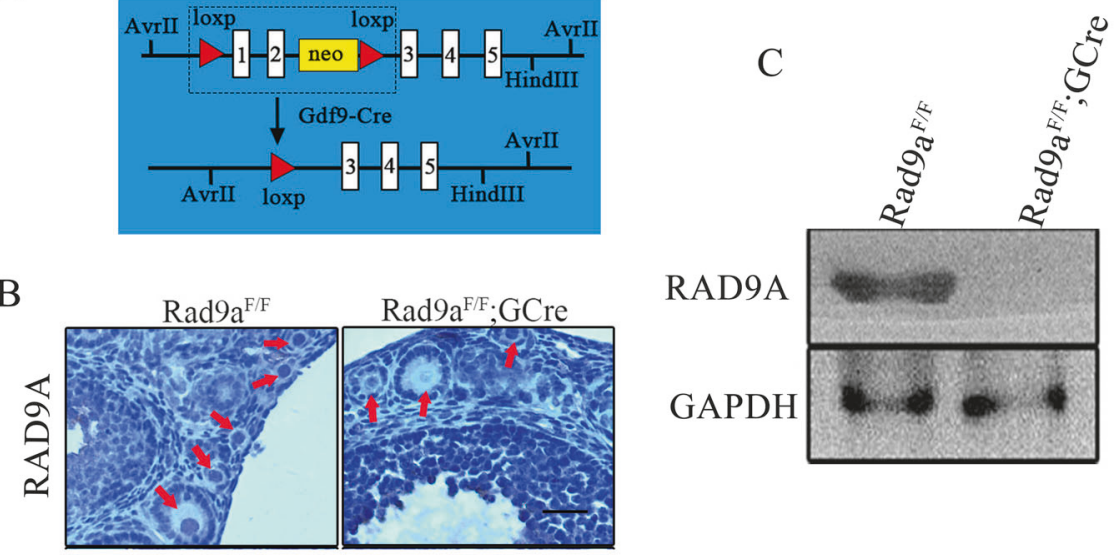

D

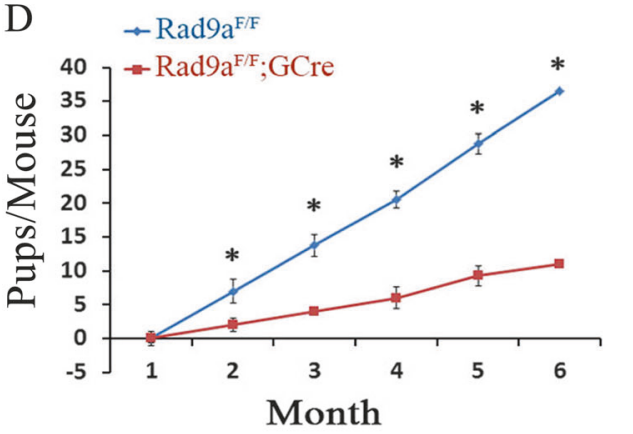

E

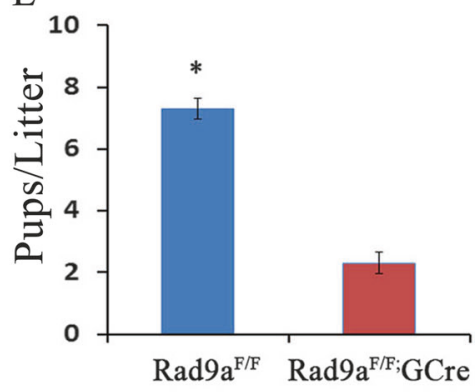

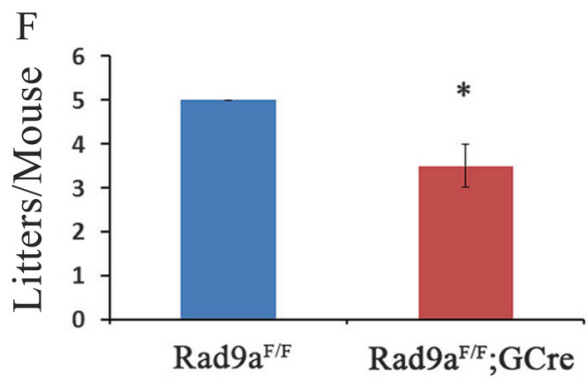

confirmed that subfertility of mutant females was attributed to post-zygotic losses, which arose from delayed chromatin decondensation. Our results determine for the first time that RAD9A is essential for zygotic chromatin reprogramming and female fertility.

\section{Results}

\section{Location of RAD9A during mouse pre-zygotic and post-zygotic embryo development}

To understand the function of RAD9A, we first characterized its expression pattern in ovaries, oocytes, and embryos of wild-type mice. RAD9A was located in the oocyte nuclei during folliculogenesis in the ovary. The signal of RAD9A was especially strong in the oocyte nuclei of primordial follicles and primary follicles. Little RAD9A staining was observed in the granulosa cells, possibly because the expression of RAD9A was below the detection limit of the assay (Fig. 1a). We also detected the expression of RAD9A during the meiotic maturation of oocytes and embryonic development by confocal microscopy, and showed that RAD9A concentrated in the oocyte nuclei at the germinal vesicle (GV) stage and disappeared after GV breakdown (GVBD) (Fig. 1b). RAD9A was relocated in the nuclei of zygote and two-cell embryos (Fig. 1c). To further confirm the nuclear localization of RAD9A, we also constructed an exogenous myc-tagged RAD9A mRNA and injected it into the oocytes, which showed the same nuclear localization of RAD9A in meiotic oocytes (Fig. 1d). 
Fig. 3 There are no evidences of pre-zygotic losses after oocytespecific ablation of RAD9A. a Representative H\&E staining of ovaries from adult female mice of both genotypes. CL, corpus luteum. b The oocytes ovulated by $\operatorname{Rad} 9 a^{F / F} ;$ GCre female mice were as normal as those of $\operatorname{Rad} 9 a^{F / F}$ mice. Bar, $100 \mu \mathrm{m}$. c Normal spindle organization in the oocytes of both genotypes. Bar, $20 \mu \mathrm{m}$. d Samples of chromosome spread of oocytes from female mice of both genotypes
A

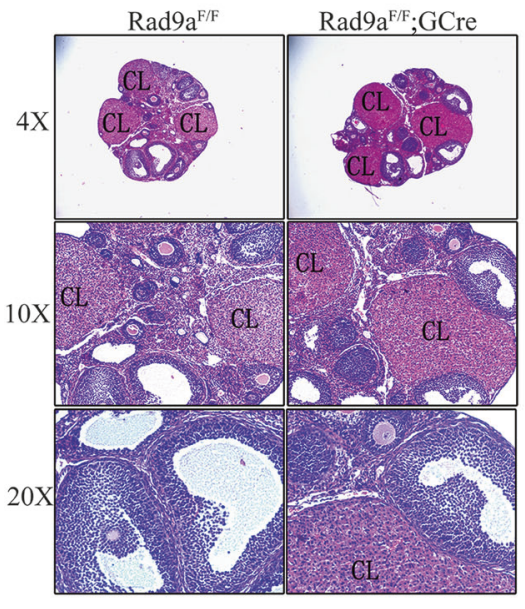

B
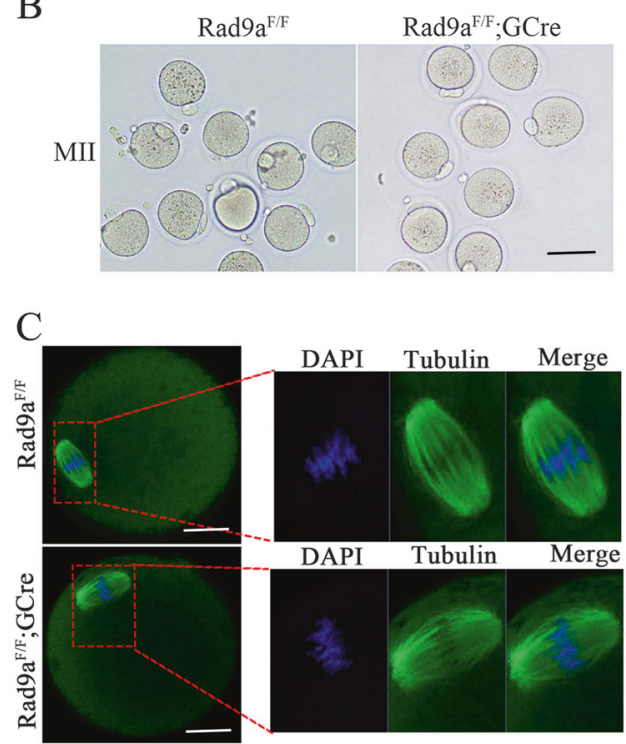

$\mathrm{D}$

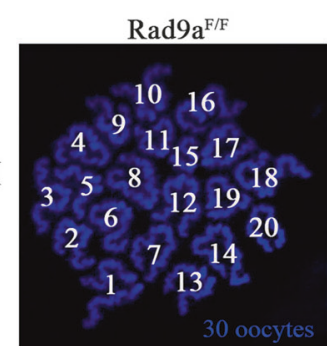

$\operatorname{Rad} 9 \mathrm{a}^{\mathrm{F} / \mathrm{F}} ; \mathrm{GCre}$

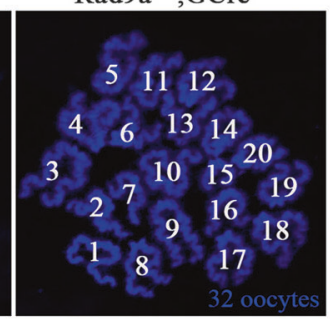

\section{Oocyte-specific disruption of RAD9A causes severe subfertility of female mice}

Because global knockout of $\operatorname{Rad} 9 a$ in mice causes embryonic lethality, it is impossible to study its role in folliculogenesis and reproductive activity of female mice [24]. So, we generated mice with oocyte-specific disruption of RAD9A by crossing $\operatorname{Rad} 9 a^{F / F}$ mice with GCre transgenic mice (referred to as $\operatorname{Rad} 9 a^{F / F}$; GCre mice) (Fig. 2a), in which Cre begins to be expressed in the oocytes of primordial follicles since postnatal Day $3[24,26]$. Then we compared the expression level of RAD9A between the oocytes of $\operatorname{Rad} 9 a^{F / F}$ and $\operatorname{Rad} 9 a^{F / F}$; GCre female mice, via immunochemistry and western blot. Results of immunochemistry indicated the loss of RAD9A location in the oocyte nuclei during folliculogenesis in $\operatorname{Rad} 9 a^{F / F} ; G C r e$ female mice (Fig. 2b). Western blot analysis also confirmed the oocyte-specific depletion of RAD9A in the $\operatorname{Rad} 9 a^{F / F}$; GCre female mice (Fig. 2c).

Next, we carried out a breeding assay by mating six $\operatorname{Rad} 9 a^{F / F}$ or $\operatorname{Rad} 9 a^{F / F} ;$ GCre female mice, respectively, with males of proven fertility during a 6-month period, to determine whether oocyte-specific disruption of RAD9A affected the fertility of mutant females. The statistical results indicated that the fertility of $\operatorname{Rad} 9 a^{F / F} ; G C r e$ female mice was sharply decreased. In average, $\operatorname{Rad} 9 a^{F / F} ; G C r e$ female mice gave birth to less than half of the pups that control female mice produced (Fig. 2d). The litter size of mutant female mice was much smaller than that of control females (Fig. 2e). Moreover, mutant female mice had less litters than control mice during a 6-month period (Fig. 2f).

\section{No obvious pre-zygotic losses occur in $\operatorname{Rad} 9 a^{F / F}$; GCre female mice}

To determine the reason why $\operatorname{Rad} 9 a^{F / F} ;$ GCre female mice became severely subfertile after oocyte-specific ablation of RAD9A, we examined folliculogenesis and meiotic maturation of oocytes in both $\operatorname{Rad} 9 a^{F / F}$ and $\operatorname{Rad} 9 a^{F / F} ;$;Cre adult female mice. No apparent morphological difference was observed in the ovaries of adult $R a d 9 a^{F / F}$; GCre female mice, compared with that of control mice (Fig. 3a). The oocytes ovulated by $\operatorname{Rad} 9 a^{F / F} ; G C r e$ female mice were also as normal as those of control mice (Fig. 3b), both of which had normal metaphase II (MII)-stage meiotic spindle organization indicated by confocal microscopy of tubulin (Fig. 3c). Analysis of chromosome spread demonstrated that mutant oocyte had the same number of normal 
Fig. 4 Oocyte-specific ablation of RAD9A causes post-zygotic losses, which are clearly identifiable at the preimplantation stages. a, b Much less but morphologically normal implantation sites in $\operatorname{Rad} 9 a^{F / F}$; GCre female mice in comparison with $\operatorname{Rad} 9 a^{F / F}$ mice, as determined by trypan blue injection on day 5. IS, implantation site; bar, $10 \mathrm{~mm}$; *, $P<0.05$. c, d Significantly fewer embryos collected from Rad $9 a^{F /}$ ${ }^{F}$; GCre female mice reached two-cell stage as compared with that from $\operatorname{Rad} 9 a^{F / F} ;$ GCre female mice. Bar, $100 \mu \mathrm{m}$. *, $P<0.05$. e, f Most of the embryos from mutant mice were arrested at zygote stage after 1-day culture. Bar, $100 \mu \mathrm{m} ; *, P<0.05$
A
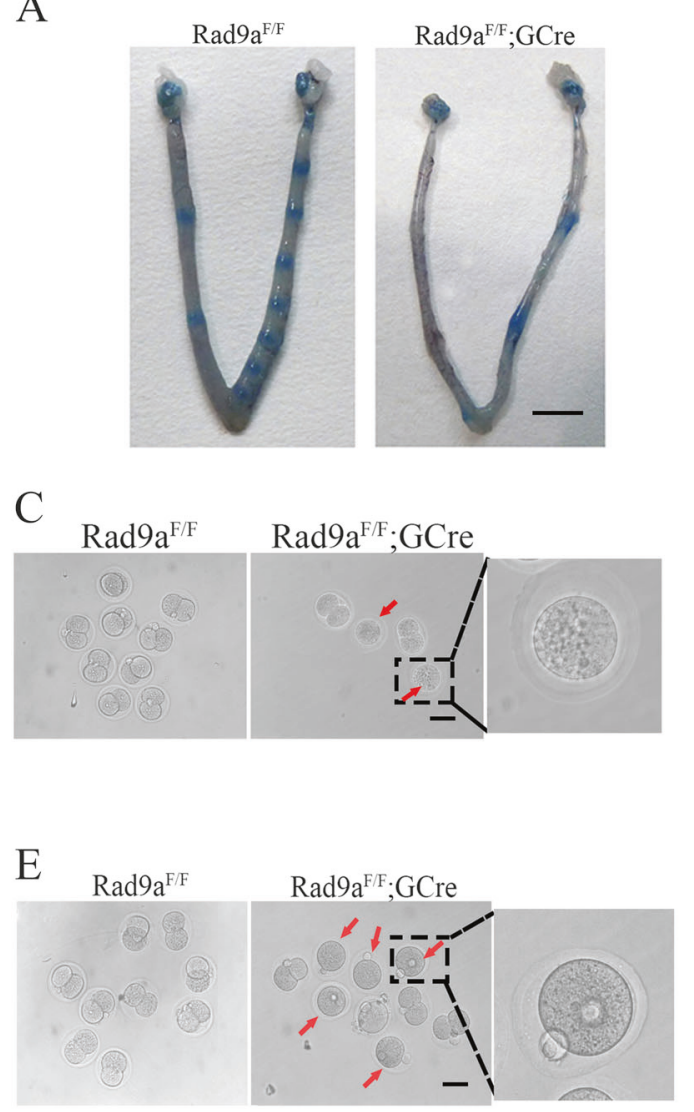

B

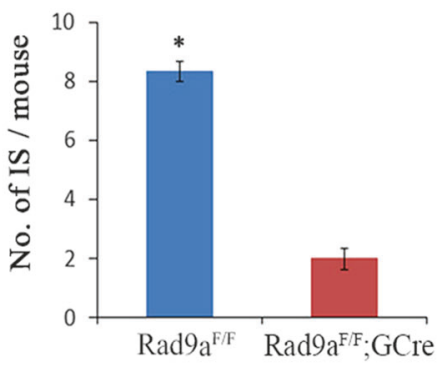

D

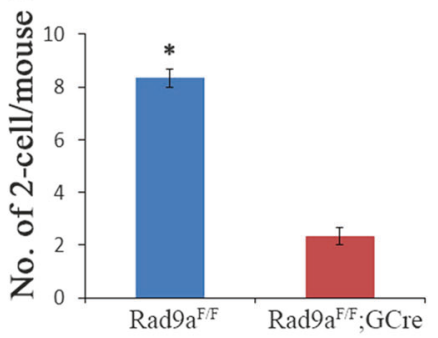

F

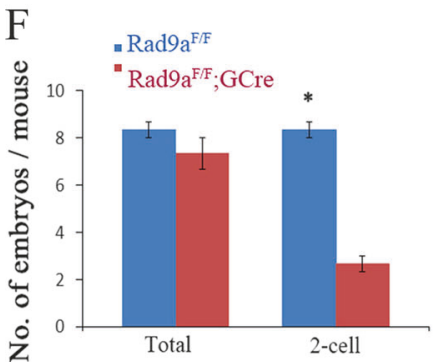

chromosomes as control oocyte (Fig. 3d). These results indicate that oocyte-specific deletion of RAD9A has no obvious impact on follicular development and meiotic maturation of oocyte. So the impairment in reproductive potential of $\operatorname{Rad} 9 a^{F / F}$; GCre female mice may come from defect in later stage of development.

\section{Subfertility of $\operatorname{Rad} 9 a^{F / F}$; GCre female mice arises from post-zygotic losses}

To further investigate the reason for subfertility of mutant mice, we crossed $\operatorname{Rad} 9 a^{F / F}$ and $\operatorname{Rad} 9 a^{F / F}$; GCre female virgin mice with wild-type males with known fertility. Mice with vagina plugs were killed after intravenous injection of trypan blue at day 5 post intercourse. Although there were no losses of pregnant corpora lutea in $\mathrm{Rad}_{9} a^{\mathrm{F} / F}$; GCre female mice (Fig S2A and S2B), the number of implantation sites in mutant mice, indicated by distinct blue bands, was much fewer than that of control mice (Fig. 4a, b). We confirmed this result via directly comparing the implanted embryos of both genotypes at day 10 post copulation (Fig S2C and S2D). More interestingly, there were more reabsorbed embryos in mutant female mice (Fig S2E and $\mathrm{S} 2 \mathrm{~F}$ ). These data illustrate that subfertility of $\operatorname{Rad} 9 a^{F / F}$; GCre female mice mainly arises from pre-implantation stage. To determine when mutant embryos started to loss, we collected embryos at day 2 post crossing from the oviducts of both genotypes with vagina plugs. All the control embryos were at the two-cell stage, whereas only a small portion of mutant embryos reached the two-cell stage, and the other portion stopped development, arrested at the zygote stage with two pronuclei (Fig. 4c, d). To further define this result, we collected zygotes at day 1 post crossing from the oviducts of both genotypes with vagina plugs for culture analysis. The number of mutant zygotes was statistically identical to that of control zygotes, indicating that ovulation and fertilization of mutant mice are normal (Fig. 4e, f). After 1-day culture, however, most of the mutant embryos were arrested at the zygotic stage, whereas all of control embryos mice reached the two-cell stage (Fig 4e, f). Moreover, most mutant oocytes are also arrested at one-cell stage after parthenogenetic activation (Figure S1A). These data indicate that reproductive defect of Rad $9 a^{F / F}$; GCre female mice arises from post-zygotic losses. 
A
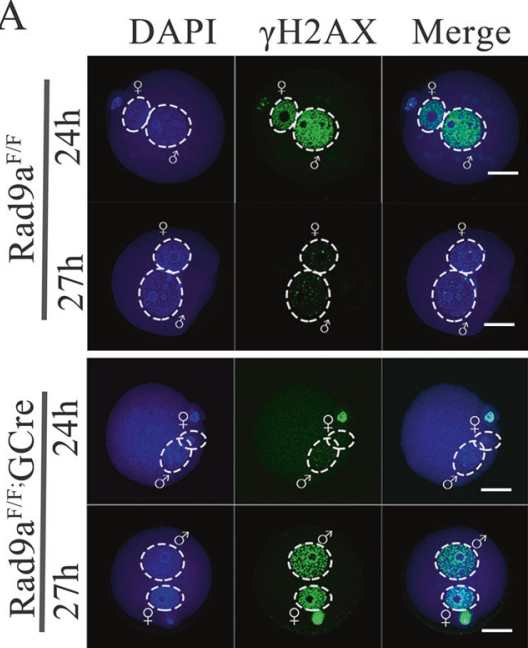

B
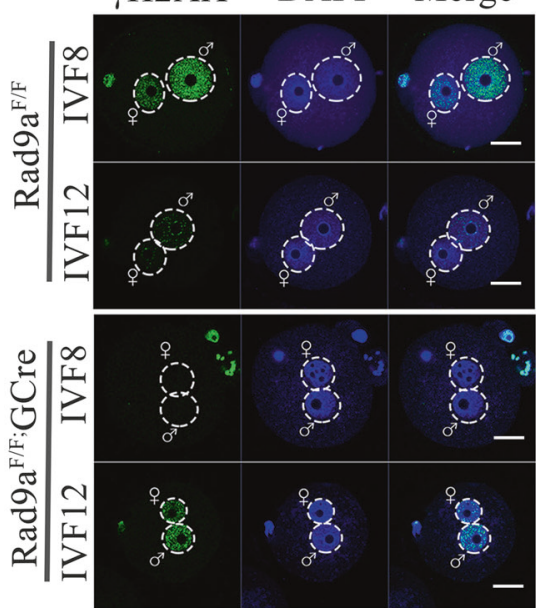

Fig. 5 Deletion of $\operatorname{Rad} 9 a$ delayes pronuclear formation and DNA replication. a Comparison of location of $\gamma \mathrm{H} 2 \mathrm{AX}$ and p-CHK2 between control and mutant zygotes derived from internal fertilization at different time points: $24 \mathrm{~h}, 24 \mathrm{~h}$ after HCG treatment; $27 \mathrm{~h}, 27 \mathrm{~h}$ after HCG treatment. Bar, $20 \mu \mathrm{m}$ b Comparison of location of $\gamma \mathrm{H} 2 \mathrm{AX}$ and p-CHK2 between control and mutant zygotes derived from IVF at different time points; IVF8, $8 \mathrm{~h}$ after IVF; IVF12, $12 \mathrm{~h}$ after IVF; bar,

\section{Delayed pronuclear formation and DNA replication in mutant zygotes triggers G2/M checkpoint}

As the well-known function of RAD9A in DNA damage response, RAD9A has a key role in G2/M checkpoint exit after replication [7, 8, 28]. Arrest of zygote development without maternal RAD9A may arise from disturbed G2/M checkpoint exit after replication. therefore, we compared the expression of phosphorylated CDC2 (p-CDC2), the key kinase promoting mitotic entry, in the zygotes of both genotypes [29]. We observed little p-CDC2 in mutant zygotes, compared with that in control $12 \mathrm{~h}$ after in vitro fertilization (IVF) (Figure S1B). Then we detected DNA repair molecules in zygotes from both in vivo fertilization

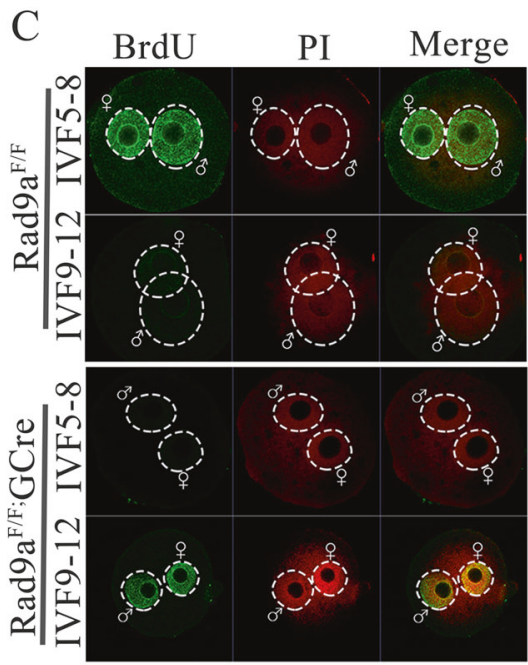

$\mathrm{D}$

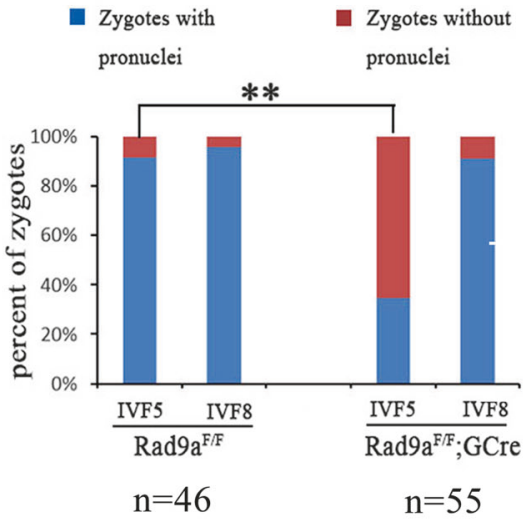

$20 \mu \mathrm{m}$. c Comparison of DNA replication between control and mutant zygotes derived from $I V F$ via BrdU labeling. IVF5-8, labeling from $5 \mathrm{~h}$ to $8 \mathrm{~h}$ after IVF; IVF9-12, labeling from $9 \mathrm{~h}$ to $12 \mathrm{~h}$ after IVF; bar, $20 \mu \mathrm{m}$. d Respective calculation of pronuclear formation for control and mutant zygotes derived from IVF. IVF5, $5 \mathrm{~h}$ after IVF; IVF8, $8 \mathrm{~h}$ after IVF; $*, P<0.05$

and IVF. Our results confirmed that localization of $\gamma \mathrm{H} 2 \mathrm{AX}$ and p-Chk2 was significantly delayed in mutant zygotes from in vivo fertilization (Fig. 5a). In addition, localization of $\gamma \mathrm{H} 2 \mathrm{AX}$ and p-Chk2 to the pronuclei of most $\operatorname{Rad} 9 a^{F / F}$; GCre zygotes was also delayed, compared with that of $\operatorname{Rad} 9 a^{F / F}$ zygotes from IVF (Fig. 5b).

The data above indicate that DNA replication in the mutant zygotes may be delayed. Indeed, as shown by the results of bromodeoxyuridine (BrdU) labeling (Fig. 5c), there was strong DNA replication activity in control zygotes from $5 \mathrm{~h}$ to $8 \mathrm{~h}$ after IVF, whereas no DNA replication activity could be observed in control zygotes from $9 \mathrm{~h}$ to $12 \mathrm{~h}$ after IVF. By contrast, there was no DNA replication activity in most of $\operatorname{Rad} 9 a^{F / F} ;$ GCre zygotes from $5 \mathrm{~h}$ to $8 \mathrm{~h}$ 
Fig. 6 Ablation of RAD9A protein disturbs chromatin decondensation in most zygotes. a Statistical calculation of zygotes of both genotypes with chromosome or pronuclei at $4 \mathrm{~h}$ after IVF. CP, condensed pronuclei; DP, decondensed pronuclei; *, $P<0.05$.

b Representative image for H3K27me1 immunostaining in zygotes of both genotypes at $4 \mathrm{~h}$ after IVF. CP, condensed pronuclei; DP, decondensed pronuclei; IVF $4 \mathrm{~h}, 4 \mathrm{~h}$ after IVF; bar, $20 \mu \mathrm{m}$. c Statistical calculation of zygotes of both genotypes with chromosome or pronuclei at $2.5 \mathrm{~h}$ after ICSI. CP, condensed pronuclei; DP, decondensed pronuclei; *, $P<$ 0.05 . ICSI $2.5 \mathrm{~h}, 2.5 \mathrm{~h}$ after ICSI; d Representative image for $\mathrm{H} 3$ immunostaining in zygotes of both genotypes at 2.5 $\mathrm{h}$ after ICSI. ICSI $2.5 \mathrm{~h}, 2.5 \mathrm{~h}$ after ICSI; bar, $20 \mu \mathrm{m}$. e chromosome segregation in zygotes of both genotypes $2.5 \mathrm{~h}$ after parthenogenetic activation. PA $2.5 \mathrm{~h}, 2.5 \mathrm{~h}$ after parthenogenetic activation
A

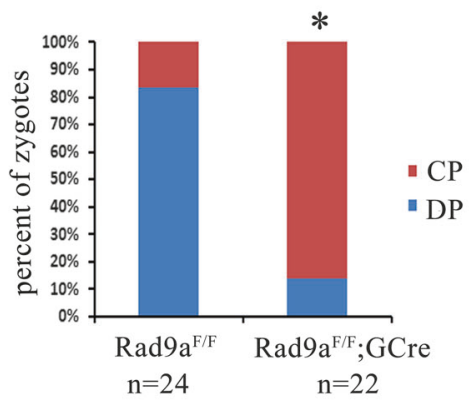

C

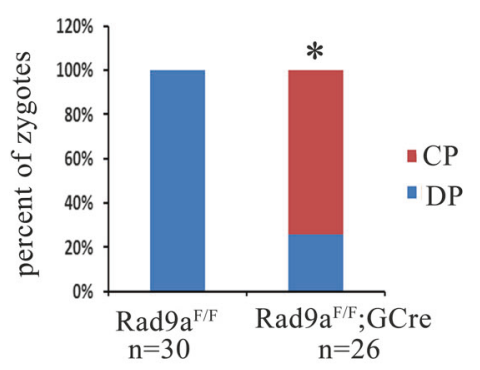

D

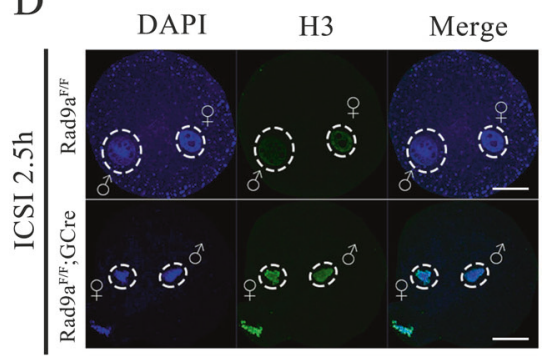

B

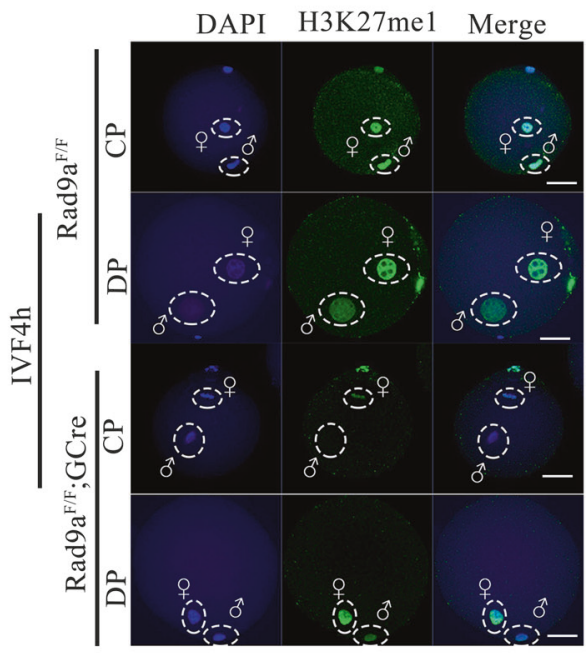

E

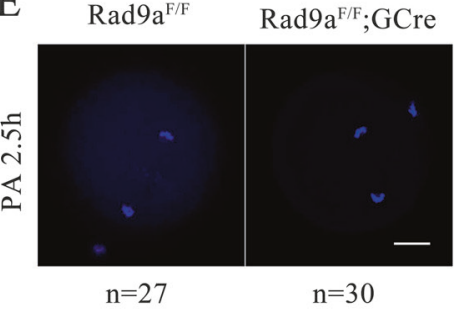

after IVF, but strong DNA replication activity could be observed in mutant zygotes from $9 \mathrm{~h}$ to $12 \mathrm{~h}$ after IVF. Delayed DNA replication may arise from disturbed pronuclear formation. To ascertain this question, we detected the development of zygotes of both genotypes derived from IVF. As shown in Fig. 5d, explicit pronuclei could be observed in majority of $\operatorname{Rad} 9 a^{F / F}$ zygotes under light microscope $5 \mathrm{~h}$ after IVF, and the number of $\operatorname{Rad} 9 a^{F / F}$ zygotes with explicit pronuclei kept constant $8 \mathrm{~h}$ after IVF. However, only a few $\operatorname{Rad} 9 a^{F / F}$; GCre zygotes with explicit pronuclei could be observed $5 \mathrm{~h}$ after IVF, and pronuclei became explicit in dominant $R a d 9 a^{F / F} ; G C r e$ zygotes $8 \mathrm{~h}$ after IVF. These data above demonstrate that deletion of maternal RAD9A delays pronuclear formation and DNA replication in zygotes, triggering G2/M checkpoint of mutant zygotes.

\section{Chromatin decondensation is delayed in most mutant zygotes}

To further examine why pronuclear formation and DNA replication are delayed in mutant zygotes after deletion of maternal RAD9A protein, we collected zygotes of both genotypes $4 \mathrm{~h}$ after IVF and compared the formation of their pronuclei via 4',6-diamidino-2-phenylindole (DAPI) staining. As shown in Fig. 6a, b, round decondensed pronuclei had been formed in most of the control zygotes $4 \mathrm{~h}$ after IVF. However, parental chromatin in most of mutant zygotes still kept condensed $4 \mathrm{~h}$ after IVF. Moreover, we also collected zygotes of both genotypes $2.5 \mathrm{~h}$ after intracytoplasmic sperm injection (ICSI). Then we compared chromatin structure in them. As shown in Fig. 6c, parental chromatins in most of mutant zygotes also kept condensed $2.5 \mathrm{~h}$ after ICSI. Meanwhile, pronuclei had been formed in the control zygotes $2.5 \mathrm{~h}$ after ICSI. This demonstrated that deferred chromatin decondensation in mutant zygotes was not attributed to delayed fertilization. Meanwhile, chromosome segregation in mutant oocytes was also normal, compared with that in control $2.5 \mathrm{~h}$ after parthenogenetic activation (Fig. 6e), i.e., deletion of maternal RAD9A had no impact on resumption of secondary meiosis of mutant oocytes. Moreover, incorporation of histone $\mathrm{H} 3$ in mutant zygotes was as normal as that in control (Fig. 6d). In all, the data above confirm that deletion of maternal RAD9A protein disturbs the chromatin decondensation in most mutant zygotes after fertilization. 
As chromatin decondensation in zygotes is accompanied with epigenetic modifications of histones [30-32], we compared the methylation of $\mathrm{H} 3$ histone in the zygotes from both genotypes. As shown in our result, strong H3K27 monomethylation (H3K27me1) signals were located at both pronuclei in all control zygotes $4 \mathrm{~h}$ after IVF (Fig. 6b). However, little H3K27me1 signals could be observed at condensed paternal pronulei, whereas strong $\mathrm{H} 3 \mathrm{~K} 27 \mathrm{me} 1$ signals were located at condensed maternal pronuclei in the mutant zygotes $4 \mathrm{~h}$ after IVF (Fig. 6b). In addition, strong signals were located at both decondensed pronulei in small part of mutant zygotes $4 \mathrm{~h}$ after IVF (Fig. 6b). Moreover, H3K27 dimethylation (H3K27me2) signals started to become apparent in paternal pronuclei of control zygotes, whereas H3K27me2 signals kept strong in maternal pronuclei $12 \mathrm{~h}$ after IVF. By comparison, almost no H3K27me2 signals could be detected in both pronuclei of mutant zygotes $12 \mathrm{~h}$ after IVF (Figure S1C). There were also almost no H3K27 trimethylation (H3K27me3) signals in both pronuclei of mutant zygotes, compared with apparent H3K27me3 signals in paternal pronuclei and strong H3K27me3 signals in maternal pronuclei of control zygotes $12 \mathrm{~h}$ after IVF (Figure S1D). Our data demonstrate that some epigenetic modifications of $\mathrm{H} 3$ histone are disturbed, which may account for delayed chromatin decondensation in most mutant zyogtes.

\section{Discussion}

As a member of 9-1-1 complex, mammalian RAD9A participates in multiple cellular processes [12]. And its role in cellular response to DNA repair in mitosis has been well established [7, 8, 13, 14]. In addition, we recently confirmed that RAD9A is required for spermatogonia differentiation [15]. However, role of RAD9A in the fertility of female mice and the corresponding mechanisms have not been demonstrated till now. In this study, we verify the importance of maternal RAD9A for zygotic development, via a conditional Rad9a knockout mouse model.

Our data reveal that RAD9A is localized in the nuclei of oocytes, zygotes and two-cell embryos. And oocyte-specific disruption of RAD9A evidently reduces the fertility of females; however, there are no evidences of pre-zygotic losses after oocyte-specific ablation of RAD9A. This result seems surprising considering the function of RAD9A in promoting genome integrity. It may be attribute to other molecules that keep genome intact during folliculogenesis and oocyte maturation after oocyte-specific ablation of RAD9A. Moreover, endogeneous DSBs in mouse oocytes mainly occur during DNA synthesis and homologous recombination, both have been finished before birth [33]. So, it is expectable that oocyte-specific ablation of RAD9A after birth by GCre has little effect on the folliculogenesis and oocyte maturation.

Strikingly, our data indicate that the reduced fertility of mutant female mice in this study is attributed to postzygotic losses. Although no defects in fertilization and secondary meiosis were observed, we confirm that arrest of mutant zygotes arises from disturbed chromatin reprogramming, i.e., deletion of maternal RAD9A delays chromatin decondensation. Pronucleus formation and DNA replication of most mutant zygotes are therefore deferred, which subsequently trigger the G2/M checkpoint and arrest development of most mutant zygotes. Our data verify the role of RAD9A in zygotic chromatin reprogramming for the first time. It is a novel discovery as previous studies focus on the role of Rad9a gene in DNA damage repair [34]. It has been reported that inactivated RAD9A delays the appearance and disappearance of ionizing radiation-induced $\gamma \mathrm{H} 2 \mathrm{AX}$ foci in cell lines $[13,14]$. We also observed this phenotype in mutant zygote, and our data provided an explicit explanation for it. Moreover, our data also verify the importance of drastic structure transformation of parental genome in zygote development after fertilization, in accordance with a recent study [6]. Previous study has indicated that disrupted incorporation of $\mathrm{H} 3$ interrupts pronuclear formation and zygote development [5, 30]. Although pronucleus has appeared in most control zygotes, parental genomes of most mutant zygotes still stay at chromosome stage. And deletion of maternal RAD9A has no impact on incorporation of $\mathrm{H} 3$ into paternal genome in our study. So zygote arrest here does not arise from interfered incorporation of $\mathrm{H} 3$, but merely from retarded chromatin decondensation. More interestingly, most mutant oocytes are also arrested at one-cell stage after pathenogenetic activation. It implies that retarded decondensation of maternal chromosomes also plays an important role in arrest of mutant zygotes. More interestingly, we detected disturbed appearance of $\mathrm{H} 3 \mathrm{~K} 27 \mathrm{me} 1, \mathrm{H} 3 \mathrm{~K} 27 \mathrm{me} 2$, and H3K27me3 in the mutant zygotes. However, more details are needed to elucidate how deletion of maternal RAD9A defers chromatin decondensation in zygotes after fertilization.

We observed that a few mutant embryos could overcome one-cell stage, and develop to post-implantation embryos, even alive offspring. This may be attributed to knockout efficiency driven by $\mathrm{Gdf} 9$-Cre, so we performed genotyping of the pups born by $\operatorname{Rad} 9 a^{F / F} ; G C r e$ female mice. Similar to a previous study [35], our results demonstrated that all the pups of mutant female mice carrying a Rad $9 a$ deletion allele. Therefore, the deletion of Rad9a gene in mutant oocytes was complete. Moreover, some mutant oocytes after pathenogenetic activation can also overcome one-cell stage. So paternal Rad9a gene has no impact on zygote development, no matter whether it is expressed in later stage 
of mutant zygote. In addition, most implanted mutant embryos, in which paternal Rad9a gene has been expressed after two-cell stage, are still reabsorbed. It means expression of paternal RAD9A cannot rescue defects in most postimplantation mutant embryos, which arises from delayed zygotic chromatin reprogramming. There may be other molecules to commit similar function to RAD9A in zygote after fertilization, which needs further study.

In summary, our findings reveal for the first time that maternal RAD9A is essential for chromatin reprogramming in mouse zygotes. Ablation of maternal RAD9A causes severe post-zygotic losses, and finally reduces fertility in mice.

\section{Materials and methods}

\section{Mice}

$\operatorname{Rad} 9 a^{\text {flox/flox }}$ mice (JAX Lab, referred to as $\operatorname{Rad} 9 a^{F / F}$ ) were maintained with a mixed genomic background of 129S4/ SvJaeand C57/BL6, and Gdf9-Cre mice were maintained with a C57/BL6 genomic background [24, 26, 27]. Mice with specific ablation of $\operatorname{Rad} 9 a$ (referred to as $\operatorname{Rad} 9 a^{F / F}$; GCre mice) were generated by crossing $\operatorname{Rad} 9 a^{F / F}$ mice with GCre mice. Mice were housed under controlled environmental conditions with free access to water and food, and 12-hour alternating light/dark cycles. Animal care and handling were conducted according to the guidelines of the Animal Research Committee of the Institute of Zoology, Chinese Academy of Sciences, China.

\section{Oocyte collection and culture}

The GV-stage oocytes were isolated from ovaries of 6- to 9week-old female mice and cultured in M2 medium under paraffin oil at $37{ }^{\circ} \mathrm{C}$ and $5 \% \mathrm{CO}_{2}$ in air. Then they were collected at different times of culture for immunofluorescent staining, western blot analysis, and microinjection.

\section{Natural ovulation, superovulation, and implantation analysis}

Virginal female mice about 2-month old were mated with fertile wild-type males to produce pregnancy and mice with vaginal plugs the next day were regarded on day 1 of pregnancy. For natural ovulation analysis, fertilized eggs were harvested from the oviducts on day 1 or day 2 , cultured, and counted. To examine implantation, pregnant female mice were injected intravenously with trypan blue and killed on day 5. And the number of implantation sites indicated by distinct blue bands was recorded. We also compared the number of implantation sites in pregnant female mice of both genotypes directly on day 10 .

To induce ovulation and collect MII oocytes, each female mouse was injected with five international units of pregnant mare's serum gonadotropin (PMSG) followed by five international units of human chorionic gonadotropin (HCG) at $48 \mathrm{~h}$ to promote ovulation. Mice were killed at 12-14 h after the HCG treatment, and cumulus-oocyte complexes were recovered from each oviduct. Cumulus-free oocytes were obtained after a 5-min treatment with hyaluronidase $(1 \mathrm{mg} / \mathrm{ml})$ in M2 medium (Sigma).

\section{In vitro and in vivo fertilization}

IVF was performed as described before with minor modifications [32]. In brief, cauda epididymis spermatozoa of males with known fertility were preincubated in human tubal fluid (HTF) medium for $1 \mathrm{~h}$ in an atmosphere of $5 \%$ $\mathrm{CO}_{2}$ and $95 \%$ air at $37{ }^{\circ} \mathrm{C}$. Superovulated cumulus-oocyte complexes were collected and inseminated with capacitated spermatozoa. After $2 \mathrm{~h}$ of insemination, fertilized eggs were washed and transferred to equilibrated KSOM + AA medium (Millipore) for further culture and analysis.

For in vivo fertilization, female mice after treatment with PMSG and HCG were crossed with fertile wild-type males. Fertilized eggs were recovered from mice with vaginal plugs the next day, at $16 \mathrm{~h}$ after the HCG treatment. Fertilized eggs were cultured and collected at the time points indicated.

\section{ICSI and parthenogenesis}

ICSI was carried out as described [36, 37]. In brief, processed spermatozoa were injected into MII-stage oocytes via an inverted microscope equipped with a Piezo-drill micromanipulation system. Fertilized eggs were transferred to equilibrated KSOM + AA medium (Millipore) for further culture and analysis.

For parthenogenesis, MII-stage oocytes were activated in calcium-free CZB medium containing $10 \mathrm{mM}$ strontium and $5 \mu \mathrm{g} / \mathrm{ml}$ cytochalasin B for 2 to $6 \mathrm{~h}$. And then embryos were collected or transferred into $\mathrm{KSOM}+\mathrm{AA}$ medium for further culture.

\section{CDNA cloning, mRNA synthesis, and microinjection}

The full-length coding sequence of mouse RAD9A was amplified from oocyte cDNA derived as described previously [38] using the following primer: Forward: 5'-T ATGGCCGGCCGTCGTGTGCAAAACAAGCTCGA-3'; Reverse: 5'-TAAGGCGCGCCGTCTAAGGACTGACGC CCAACTC- $3^{\prime}$. The PCR products were purified, then 
digested using FseI and AscI (New England Biolabs, Inc.) and cloned into the pCS2 + vector. The synthesis and microinjection of myc-tagged RAD9A mRNA were performed as described previously [39].

\section{Antibodies, immunofluorescence, and confocal microscopy}

Antibodies used in this study were purchased from the following companies: mouse monoclonal anti-RAD9A (BD Biosciences, USA); rabbit polyclonal anti $\gamma \mathrm{H} 2 \mathrm{AX}$ (Cell Signaling Technology, Beverly, MA, USA); rabbit polyclonal anti p-CHK2 (T68) (Bioworld, MN, USA); mouse monoclonal anti $\alpha$-Tubulin-FITC (Sigma); anti Myc-FITC and anti GAPDH (Invitrogen, Carlsbad, CA, USA); mouse monoclonal anti H3(EMAR, Beijing, China); rabbit polyclonal anti H3K27me1(Abcam, Cambridge, UK); rabbit polyclonal anti H3K27me2(Cell Signaling Technology, Beverly, MA, USA); rabbit monoclonal anti H3K27me3 (Cell Signaling Technology, Beverly, MA, USA); rabbit polyclonal anti p-CDC2 (ABclonal, Beverly, MA, USA); mouse monoclonal anti ACTIN (ZhongShan Golden Bridge Biotechnology, Beijing, China); Secondary antibodies were purchased from ZhongShan Golden Bridge Biotechnology Co., Ltd (Beijing, China). Immunofluorescent analysis and confocal microscopy were performed as described previously [40] using the following antibody dilution: antiRAD9A/ p-Chk2/myc-FITC/H3/ H3K27me1/H3K27me2/ H3K27me3/ $\alpha$-tubulin-FITC: 1:200 and anti $\gamma \mathrm{H} 2 \mathrm{AX}: 1: 400$.

\section{Chromosome spread}

For chromosome spread, MII oocytes were left in hypotonic solution (1\% Na Citrate) for $20 \mathrm{~min}$ at room temperature and fixed with methanol/glacial acetic acid (3:1). The oocytes were then stained with DAPI for $5 \mathrm{~min}$ and slides were mounted for observation by immunofluorescence microscopy as described previously [41].

\section{Histological analysis and immunohistochemistry}

Ovaries were collected from adult female mice, fixed in $4 \%$ paraformaldehyde overnight at $4^{\circ} \mathrm{C}$, dehydrated in a graded ethanol series, cleared in xylene, and embeded in paraffin. Then $8 \mu \mathrm{m}$ serial sections are prepared for hematoxylin and eosin analysis. Immunohistochemistry was also performed on $8 \mu \mathrm{m}$ sections using the Vectastain $\mathrm{ABC}$ kit (Vector Laboratories, Burlingame, CA). The anti-RAD9A antibody dilution was 1:200.

\section{BrdU labeling for DNA replication}

BrdU labeling for DNA replication test was performed as described previously with minor modifications. In short, the zygotes were incubated in HTF medium supplemented with $100 \mu \mathrm{M}$ BrdU (858811; Sigma-Aldrich) in an atmosphere of $5 \% \mathrm{CO} 2$ and $95 \%$ air at $37{ }^{\circ} \mathrm{C}$. After incubation, the zygotes were fixed for $15 \mathrm{~min}$ in $4 \%$ paraformaldehyde in phosphate-buffered saline (PBS), permeabilized with $0.5 \%$ Triton X-100 in PBS for $20 \mathrm{~min}$ at room temperature, treated with $4 \mathrm{~N} \mathrm{HCl}$ at room temperature for $10 \mathrm{~min}$, and subsequently neutralized for $10 \mathrm{~min}$ with $100 \mathrm{mM}$ Tris-HCl buffer ( $\mathrm{pH} 8.5$ ) after permeabilization. After blocking at $4{ }^{\circ}$ $\mathrm{C}$ overnight with $0.2 \%$ Tween 20 in PBS/bovine serum albumin (BSA; PBS containing $10 \mathrm{mg} / \mathrm{ml}$ BSA), the cells were incubated with anti-BrdU antibody (1:1000; B8434; Sigma-Aldrich), followed by secondary Alexa Fluor 488-conjugated antibody (1:1000; A11001; Life Technologies, Shanghai, China), according to the procedure of confocal microscopy.

\section{Western blot analysis}

A total of 150 mouse oocytes or zygotes per sample were mixed with sodium lauryl sulfate sample buffer and boiled for $5 \mathrm{~min}$ at $100{ }^{\circ} \mathrm{C}$ for sulfate polyacrylamide gel electrophoresis. Western blots were performed as described previously [42] using the following antibody dilution: antiRAD9A/GAPDH: 1:1000; p-CDC2/ACTIN: 1:1000.

\section{Statistical analysis}

All experiments were repeated at least three times for statistical analysis. For comparisons, means and standard deviations were calculated, and the difference between two groups was analyzed with Student's $t$ test or $\chi^{2}$-test. Difference was considered statistically significant if $P<0.05$.

Acknowledgements We are grateful to Shi-Wen Li, Li-Juan Wang, and Hua Qin for their technical assistance. We also thank Dr. Heng-Yu Fan for providing antibodies, Dr. Enkui Duan, Dr. Ying Zhang, Dr. Zhi-Ming Han, and Dr. Jing-Pian Peng for their useful suggestions. This study was supported by the National Key R\& D Program of China (2016YFA0100400, 2016YFC1000600) and the National Natural Science Foundation of China (No. 31272260, 31371451, 31530049).

Author contributions L.H. and Q.-Y.S. conceived and designed the experiments. L.H., T.-G.M., X.-S.M., Z.-B.W., S.-T.Q., Q.C., Q.-H.Z., Q.-X.L., Z.-W.W., M.-W.H., L.G., and Y.-C.O. performed the experiments. L.H. analyzed the data. Y.H. contributed reagents, 
materials and analysis tools. Y.Z. provided the $\operatorname{Rad} 9 \mathrm{a}^{\mathrm{F} / \mathrm{F}}$ mouse strain. L.H. and Q.-Y.S. wrote the manuscript. All authors read and approved the final manuscript.

\section{Compliance with ethical standards}

Conflict of interest The authors declare that they have no conflict of interest.

\section{References}

1. Clift D, Schuh M. Restarting life: fertilization and the transition from meiosis to mitosis. Nat Rev Mol Cell Biol. 2013;14:549-62.

2. Cabot B, Cabot RA. Chromatin remodeling in mammalian embryos. Reproduction. 2018;155:R147-R158.

3. Gu TP, Guo F, Yang H, Wu HP, Xu GF, Liu W, et al. The role of Tet3 DNA dioxygenase in epigenetic reprogramming by oocytes. Nature. 2011;477:606-10.

4. Yamanaka S, Blau HM. Nuclear reprogramming to a pluripotent state by three approaches. Nature. 2010;465:704-12.

5. Lin CJ, Koh FM, Wong P, Conti M, Ramalho-Santos M. Hiramediated H3.3 incorporation is required for DNA replication and ribosomal RNA transcription in the mouse zygote. Dev Cell. 2014;30:268-79.

6. Du Z, Zheng H, Huang B, Ma R, Wu J, Zhang X, et al. Allelic reprogramming of $3 \mathrm{D}$ chromatin architecture during early mammalian development. Nature. 2017;547:232-5.

7. Delacroix S, Wagner JM, Kobayashi M, Yamamoto K, Karnitz LM. The Rad9-Hus1-Rad1 (9-1-1) clamp activates checkpoint signaling via TopBP1. Genes Dev. 2007;21:1472-7.

8. Niida H, Nakanishi M. DNA damage checkpoints in mammals. Mutagenesis. 2006;21:3-9.

9. Jekimovs C, Bolderson E, Suraweera A, Adams M, O'Byrne KJ, Richard DJ. Chemotherapeutic compounds targeting the DNA double-strand break repair pathways: the good, the bad, and the promising. Front Oncol. 2014;4:86.

10. Bashkirov VI, Bashkirova EV, Haghnazari E, Heyer WD. Direct kinase-to-kinase signaling mediated by the FHA phosphoprotein recognition domain of the Dun1 DNA damage checkpoint kinase. Mol Cell Biol. 2003;23:1441-52.

11. Lieberman HB. Rad9, an evolutionarily conserved gene with multiple functions for preserving genomic integrity. J Cell Biochem. 2006;97:690-7.

12. Griffith JD, Lindsey-Boltz LA, Sancar A. Structures of the human Rad17-replication factor $\mathrm{C}$ and checkpoint Rad 9-1-1 complexes visualized by glycerol spray/low voltage microscopy. J Biol Chem. 2002;277:15233-6.

13. Dang T, Bao S, Wang XF. Human Rad9 is required for the activation of S-phase checkpoint and the maintenance of chromosomal stability. Genes Cells. 2005;10:287-95.

14. Pandita RK, Sharma GG, Laszlo A, Hopkins KM, Davey S, Chakhparonian M, et al. Mammalian Rad9 plays a role in telomere stability, S- and G2-phase-specific cell survival, and homologous recombinational repair. Mol Cell Biol. 2006;26:1850-64.

15. Vasileva A, Hopkins KM, Wang X, Weisbach MM, Friedman RA, Wolgemuth DJ, et al. The DNA damage checkpoint protein RAD9A is essential for male meiosis in the mouse. J Cell Sci. 2013;126(Pt 17):3927-38.

16. Yin Y, Zhu A, Jin YJ, Liu YX, Zhang X, Hopkins KM, et al. Human RAD9 checkpoint control/proapoptotic protein can activate transcription of p21. Proc Natl Acad Sci USA. 2004;101:8864-9.
17. Komatsu K, Miyashita T, Hang H, Hopkins KM, Zheng W, Cuddeback S, et al. Human homologue of S. pombe Rad9 interacts with BCL-2/BCL-xL and promotes apoptosis. Nat Cell Biol. 2000;2:1-6.

18. Lee MW, Hirai I, Wang HG. Caspase-3-mediated cleavage of Rad9 during apoptosis. Oncogene. 2003;22:6340-6.

19. Huang L, Wang ZB, Qi ST, Ma XS, Liang QX, Lei G, et al. Rad9a is required for spermatogonia differentiation in mice. Oncotarget. 2016;7:86350-8.

20. Zhu A, Zhang CX, Lieberman HB. Rad9 has a functional role in human prostate carcinogenesis. Cancer Res. 2008;68: 1267-74.

21. Broustas CG, Lieberman HB. Contributions of Rad9 to tumorigenesis. J Cell Biochem. 2012;113:742-51.

22. Hu Z, Liu Y, Zhang C, Zhao Y, He W, Han L, et al. Targeted deletion of Rad9 in mouse skin keratinocytes enhances genotoxininduced tumor development. Cancer Res. 2008;68: 5552-61.

23. Cheng CK, Chow LW, Loo WT, Chan TK, Chan V. The cell cycle checkpoint gene $\operatorname{Rad} 9$ is a novel oncogene activated by $11 \mathrm{q} 13$ amplification and DNA methylation in breast cancer. Cancer Res. 2005;65:8646-54.

24. Hopkins KM, Auerbach W, Wang XY, Hande MP, Hang H, Wolgemuth DJ, et al. Deletion of mouse rad9 causes abnormal cellular responses to DNA damage, genomic instability, and embryonic lethality. Mol Cell Biol. 2004;24:7235-48.

25. Kleiman NJ, David J, Elliston CD, Hopkins KM, Smilenov LB, Brenner DJ, et al. Mrad9 and atm haploinsufficiency enhance spontaneous and X-ray-induced cataractogenesis in mice. Radiat Res. 2007;168:567-73.

26. Lan ZJ, Xu X, Cooney AJ. Differential oocyte-specific expression of Cre recombinase activity in GDF-9-iCre, Zp3cre, and Msx2Cre transgenic mice. Biol Reprod. 2004;71:1469-74.

27. Hu MW, Wang ZB, Schatten H, Sun QY. New understandings on folliculogenesis/oogenesis regulation in mouse as revealed by conditional knockout. J Genet Genom. 2012;39:61-68.

28. Smilenov LB, Lieberman HB, Mitchell SA, Baker RA, Hopkins KM, Hall EJ. Combined haploinsufficiency for ATM and RAD9 as a factor in cell transformation, apoptosis, and DNA lesion repair dynamics. Cancer Res 2005;65:933-8.

29. Yan L, Donze JR, Liu L. Inactivated MGMT by O6benzylguanine is associated with prolonged $\mathrm{G} 2 / \mathrm{M}$ arrest in cancer cells treated with BCNU. Oncogene. 2005;24:2175-83.

30. Loppin B, Bonnefoy E, Anselme C, Laurencon A, Karr TL, Couble P. The histone H3.3 chaperone HIRA is essential for chromatin assembly in the male pronucleus. Nature. 2005;437:1386-90.

31. Santos F, Peters AH, Otte AP, Reik W, Dean W. Dynamic chromatin modifications characterise the first cell cycle in mouse embryos. Dev Biol. 2005;280:225-36.

32. Ma XS, Chao SB, Huang XJ, Lin F, Qin L, Wang XG, et al. The dynamics and regulatory mechanism of pronuclear $\mathrm{H} 3 \mathrm{k} 9 \mathrm{me} 2$ asymmetry in mouse zygotes. Sci Rep. 2015;5:17924.

33. Crichton JH, Playfoot CJ, Adams IR. The role of chromatin modifications in progression through mouse meiotic prophase. $\mathrm{J}$ Genet Genom. 2014;41:97-106.

34. Greer DA, Besley BD, Kennedy KB, Davey S. hRad9 rapidly binds DNA containing double-strand breaks and is required for damage-dependent topoisomerase II beta binding protein 1 focus formation. Cancer Res. 2003;63:4829-35.

35. Ma XS, Lin F, Wang ZW, Hu MW, Huang L, Meng TG, et al. Geminin deletion in mouse oocytes results in impaired embryo development and reduced fertility. Mol Biol Cell. 2016;27:768-75. 
36. Chao SB, Guo L, Ou XH, Luo SM, Wang ZB, Schatten H, et al. Heated spermatozoa: effects on embryonic development and epigenetics. Hum Reprod. 2012;27:1016-24.

37. Guo L, Chao SB, Xiao L, Wang ZB, Meng TG, Li YY, et al. Sperm-carried RNAs play critical roles in mouse embryonic development. Oncotarget. 2017;8:67394-405.

38. Jiang ZZ, Hu MW, Wang ZB, Huang L, Lin F, Qi ST, et al. Survivin is essential for fertile egg production and female fertility in mice. Cell Death Dis. 2014;5:e1154.

39. Qi ST, Wang ZB, Ouyang YC, Zhang QH, Hu MW, Huang X, et al. Overexpression of SETbeta, a protein localizing to centromeres, causes precocious separation of chromatids during the first meiosis of mouse oocytes. J Cell Sci. 2013;126:1595-603.
40. Hu MW, Wang ZB, Jiang ZZ, Qi ST, Huang L, Liang QX, et al. Scaffold subunit Aalpha of PP2A is essential for female meiosis and fertility in mice. Biol Reprod. 2014;91:19.

41. Wang ZB, Jiang ZZ, Zhang QH, Hu MW, Huang L, Ou XH, et al. Specific deletion of Cdc42 does not affect meiotic spindle organization/migration and homologous chromosome segregation but disrupts polarity establishment and cytokinesis in mouse oocytes. Mol Biol Cell. 2013;24:3832-41.

42. Zhang D, Ma W, Li YH, Hou Y, Li SW, Meng XQ, et al. Intraoocyte localization of MAD2 and its relationship with kinetochores, microtubules, and chromosomes in rat oocytes during meiosis. Biol Reprod. 2004;71:740-8. 